\title{
Immune Abnormalities in Patients Meeting New Diagnostic Criteria for Chronic Fatigue Syndrome/Myalgic Encephalomyelitis
}

\author{
Brenu EW ${ }^{1,2 *}$, Johnston $\mathbf{S}^{1,2}$, Hardcastle SL ${ }^{1,2}$, Huth TK ${ }^{1,2}$, Fuller $K^{1,2}$, Ramos SB ${ }^{1,2}$, Staines DR ${ }^{2,3}$ and Marshall-Gradisnik SM1,2 \\ ${ }^{1}$ School of Medical Science, Griffith University, Gold Coast, Queensland, Australia \\ ${ }^{2}$ The National Centre for Neuroimmunology and Emerging Diseases, Griffith Health Institute, Queensland, Australia \\ ${ }^{3}$ Queensland Health, Gold Coast Public Health Unit, Robina, Gold Coast, Queensland, Australia
}

\begin{abstract}
Background: Immunological abnormalities have been identified in Chronic Fatigue Syndrome/Myalgic Encephalomyelitis patients fulfilling the 1994 Centers for Disease Control diagnostic criteria. Significant developments have been made to diagnostic criteria, but potential immunological markers have not been assessed in patients fulfilling these latest clinical requirements. Therefore, this study evaluated immunological parameters in patients that also fulfill the latest diagnostic criteria available known as the International Consensus Criteria.

Methods: The Immunological investigations including Natural Killer cell activity and phenotyping studies for dendritic cells, neutrophils, B cells and regulatory T cells were performed on whole blood samples collected from all participants using flow cytometric protocols. The physical functioning of all participants was also evaluated using scores from the Short Form Health Survey, and the World Health Organization Disability Adjustment Schedule. Results were compared according 1994 Centers of Disease Control and Prevention defined patients, and International Consensus Criteria defined patients, and healthy controls.

Results: Natural killer cell activity was consistently and significantly decreased, and regulatory $\mathrm{T}$ cells were significantly increased in both patient groups compared to healthy controls. Differences were found in human neutraphil antigens and expression of natural killer cell receptors between patient groups. Highly significant correlations were also found between physical status and some immune parameters in International Consensus Criteria defined patients.
\end{abstract}

Conclusion: This preliminary investigation on different diagnostic criteria suggests that the International Consensus Criteria may be more effective a detecting salient differences in the immune system.

Keywords: Chronic fatigue syndrome; Myalgic encephalomyelitis; Diagnosis; Immunology; Physical functioning

Abbreviations: CDC: Centres for Disease Control and Prevention; CFS: Chronic Fatigue Syndrome; HNA: Human Neutrophil Antigen; KIR: Killer Immunoglobulin Receptor; NK: Natural Killer; Tregs: Regulatory T cells; WHO DAS 2.0: World Health Organization Disability Adjustment Schedule 2.0; ICC: International Consensus Criteria; ME: Myalgic Encephalomyelitis; SF-36: Short Form Health Survey

\section{Introduction}

Chronic Fatigue Syndrome, also referred to as Myalgic Encephalomyelitis (CFS/ME) encompasses a range of symptoms in response to unknown agents and physiological processes. In clinical research settings, CFS/ME has been defined according to an array of different diagnostic criteria that include the 1988 Centers for Disease Control and Prevention (CDC), 1994 CDC, Australian, British, 2003 Canadian Consensus, and 2011 International Consensus Criteria (ICC) [1-6]. The 1994 CDC has been the most extensively used, and requires the presence of a chronic, debilitating fatigue for 6 months and at least four of following eight symptoms: post-exertional malaise, unrefreshing sleep, muscle pain, joint pain, headaches, sore throat and tender lymph nodes. In contrast, the ICC categorises symptoms according to postexertional fatigue, and dysfunction of the neurological, immunological, autonomic and metabolic systems. Accordingly, patients are required to fulfill required number of symptoms from each category. This new criteria allows for a more holistic investigation of CFS/ME, where patients exhibit unique combinations of symptoms that result in mild to severe impairment in physical functioning.
This latest criteria was introduced to incorporate the increasing evidence on physiological impairment that has been demonstrated in CFS/ME. A particularly consistent finding in CFS/ME is found in the immunological system, as Natural Killer (NK) cell activity has been identified as an important marker that is significantly reduced across all patients [7]. Increases in regulatory $\mathrm{T}$ cells (Tregs) have also been reported, while cytokines remain controversial. These findings to date however have largely been based on patients defined according to 1994 CDC criteria. The symptom requirements of the 1994 CDC are considered broadly inclusive, which may increase the likelihood of clinically heterogeneous patient sets $[8,9]$. This can be a specific source of variability in immunological findings in CFS/ME. The more specific requirements of the ICC however, may select patients that are less clinically diverse. This could improve detection of immunological abnormalities, as well consistency across research. Previously, greater impairment in physical functioning has been identified in ICC patients

*Corresponding author: Ekua Weba Brenu, Griffith University, Griffith Health Centre, National Centre for Neuroimmunology and Emerging Diseases, Queensland, Australia 4222, Tel: +61 75678 0725; E-mail: e.brenu@griffith.edu.au

Received October 24, 2013; Accepted November 12, 2013; Published November 14, 2013

Citation: Brenu EW, Johnston S, Hardcastle SL, Huth TK, Fuller K, et al. (2013) Immune Abnormalities in Patients Meeting New Diagnostic Criteria for Chronic Fatigue Syndrome/Myalgic Encephalomyelitis. J Mol Biomark Diagn 4: 152 doi:10.4172/2155-9929.1000152

Copyright: (c) 2013 Brenu EW, et al. This is an open-access article distributed under the terms of the Creative Commons Attribution License, which permits unrestricted use, distribution, and reproduction in any medium, provided the original author and source are credited 
Citation: Brenu EW, Johnston S, Hardcastle SL, Huth TK, Fuller K, et al. (2013) Immune Abnormalities in Patients Meeting New Diagnostic Criteria for Chronic Fatigue Syndrome/Myalgic Encephalomyelitis. J Mol Biomark Diagn 4: 152. doi:10.4172/2155-9929.1000152

Page 2 of 6

in comparison to $1994 \mathrm{CDC}[10,11]$. As ICC patients represent a distinct clinical group, it is important to assess whether immunological anomalies previously identified in CFS/ME are present.

Hence, the purpose of this investigation was to compare immunological parameters of CFS/ME patients that fulfill the 1994 CDC and ICC criteria, and determine if certain criteria may be more effective at identifying salient abnormalities in comparison to healthy controls. An important question regards the significance of immunological parameters in relation to physical function. This study therefore, also investigated whether significant correlations were found between measures of immune and physical status.

\section{Methods}

\section{Participants}

The study recruited 63 participants of which 41 were CFS/ME patients and 22 were healthy controls. Participants were recruited from South East Queensland from the National Centre for Neuroimmunology and Emerging Diseases database of patients diagnosed with CFS/ME. An independent researcher who was not involved in the laboratory experiments administered written consent forms and a questionnaire to be completed before blood collection. The questionnaire included a symptom checklist to ascertain diagnostic criteria CFS/ME, and also screened for exclusionary conditions that included autoimmune disorders, psychosis, epilepsy, cardiac related disorders, smoking, pregnancy or breastfeeding. To be confirmed as a CFS/ME patient, their reported symptoms had to comply with the 1994 CDC criteria or ICC.

Patients that only met the 1994 CDC criteria were classified as 1994 CDC. Patients fulfilling the ICC were also found to meet the 1994 CDC requirements, but were classified as ICC patients. CFS/ME patients that did not meet either criteria, or had exclusionary conditions were not included in the study. Healthy controls were defined as those with no symptoms of CFS/ME and no exclusionary conditions. Approval for this study was obtained from the Griffith University Human Research Ethics Committee. The code of practice for this study adhered to the ethical guidelines outlined in the 1975 Declaration of Helenski.

\section{Evaluation of physical functioning}

Prior to blood collection, all participants completed self-reporting measures of their health according to the Short Form Health Survey (SF-36) [12], and the World Health Organization Disability Adjustment Schedule (WHO DAS 2.0) [5,13]. The SF-36 and WHO DAS 2.0 provide scores for different aspects of physical and mental health; however analysis was confined to scores indicative of physical status only. Accordingly, the SF-36 score for Physical Functioning (PF), as well as the overall score for the physical health component of the survey, referred to as the Physical Component Summary (PCS) was calculated using ranges from 0 to 100 , with lower values indicating greater impairment. The WHO DAS 2.0 score for Mobility (M) was calculated ranging from 0 to 100 was also used, however higher values indicate greater impairment.

\section{Sample preparation}

Researchers were blinded to the grouping of the participants. Whole blood samples were collected from all participants into EDTA and heparinised blood tubes. Routine assessment of blood characteristics were performed prior to all laboratory experiments. With the exception of NK cell phenotyping experiments whole blood was used for all phenotyping studies. For all flow cytometer experiments, the monoclonal antibodies were fluorescently labeled with fluorescein isothiocynate, phycoerythrin, allophycocyanin and peridinin chlorophyll, and analysis was performed on a four color flow cytometer.

\section{Evaluation of NK cell activity}

The activity of NK cells was measured using a well-known flow cytometeric method [14-16]. Briefly, isolated PBMCs $\left(1 \times 10^{6}\right.$ cells $\left./ \mathrm{ml}\right)$ were stained with $0.4 \%$ PKH-26 fluorescent cell linker dye (SigmaAldrich, St Louis, MO). The target cells used were K562 tumour cells $\left(1 \times 10^{5} \mathrm{cells} / \mathrm{ml}\right)$. The effector cells and the target cells were incubated at three effector to target ratios; $12.5: 1,25: 1$ and 50:1 for four hours at $37^{\circ} \mathrm{C}$. Control samples without PBMCs were also included to determine the number of cells that had undergone necrosis. Cells were stained with annexin $\mathrm{V}$ and 7-AAD (Becton Dickinson (BD) Pharmingen, San Diego, CA) and analysed on the BD FACS Calibur flow cytometer. Percent lysis was calculated for each sample at the different ratios as described previously [14-16].

\section{Assessment of cell phenotypes}

Flurochrome conjugated monoclonal antibodies were used to determine the distribution of the phenotypes of the following cells, DCs (plasmacytoid (pDCs), myeloid (mDCs) and Cluster Of Differentiation (CD) $16^{+}$DCs), NK cells (CD56 ${ }^{\text {dim }}$ and CD56 ${ }^{\text {bright }}$ ), B cells (immature, memory and plasma) and Tregs. Cells were stained with the following monoclonal antibodies CD3, CD19, CD21, CD20, CD27, CD16, CD11c, CD62L, CD127, CD4, CD25, CD73, CD39, CD56, CD38, CD138 and CD10 (BD Pharmingen, San Diego, CA). Absolute counts for each cell subset were calculated from percentage of gated events on the flow cytometer and absolute WBCs numbers from haematological counts [17]. To determine the expression of various types of Human Neutrophil Antigen (HNA), samples were labeled with a combination of the following antibodies HNA-1 (CD16b), HNA-2 (CD177), HNA-4 (CD11b) and HNA-5 (CD11a).

\section{Intracellular staining of functional proteins}

NK lytic proteins and FOXP3 levels was assessed via intracellular staining [18]. Isolated PBMCs $\left(1 \times 10^{7} \mathrm{cells} / \mathrm{ml}\right)$ were labelled with fluorochrome-conjugated monoclonal antibodies CD56, CD16 and CD3; CD25, CD127 and CD4 (BD Pharmingen, San Diego, CA) for the seclusion of NK cells and Tregs from whole blood samples. Samples were then fixed and permeablised with BD Cytofix (BD Pharmingen, San Diego, CA) and diluted perm wash buffer (BD Pharmingen, San Diego, CA) respectively. Cells were then stained with perforin, granzyme A (GZA), granzyme B (GZB) or FOXP3 (BD Pharmingen, San Diego, $C A)$ and analysed on the flow cytometer.

\section{Examination of killer immunoglobulin receptor (KIRs) on isolated NK cells}

The expression of KIR receptors on the surfaces of NK cells was determined on isolated NK cells. Briefly, Rosettesep (Stemcells Inc, Germany) was added to whole blood samples to preferentially label all other cells with the exception of NK cells. Blood samples were then diluted in PBS and layered over ficoll hypaque. Following isolation, $\mathrm{NK}$ cells were stained with monoclonal antibodies including CD3, CD16, CD56, CD158a/h (KIR2DL1/S1), CD158e (KIR3DL1), CD158b (KIR2DL2/DL3), CD158i (KIR2DS4) (Miltenyi Biotech, Bergisch Glabach, Germany) and NKG2D (BD Bioscience, San Jose, CA) [19]. Subsequently, samples were analysed on the flow cytometer. 
Citation: Brenu EW, Johnston S, Hardcastle SL, Huth TK, Fuller K, et al. (2013) Immune Abnormalities in Patients Meeting New Diagnostic Criteria for Chronic Fatigue Syndrome/Myalgic Encephalomyelitis. J Mol Biomark Diagn 4: 152. doi:10.4172/2155-9929.1000152

Page 3 of 6

\section{Statistical analysis}

SPSS v.22 was used determine whether differences in immunological parameters between the 1994 CDC patient, ICC patient, and healthy control subgroups using independent $t$-testing with a significance at $\mathrm{p}<0.05$. To compare the relationship between immunological parameters and mean physical functioning scores for three independent groups: 1994 CDC cases, ICC cases, and healthy controls, using Spearman's correlation. Results of correlation were only considered significant at the $p<0.001$ level.

\section{Results}

\section{Diagnoses based on the 1994 CDC and ICC}

Of $41 \mathrm{CFS} / \mathrm{ME}$ patients recruited, 17 were classified as $1994 \mathrm{CDC}$, 18 were classified as ICC, and 5 did not meet inclusion criteria. All 22 healthy controls met the study inclusion criteria. Mean age was similar

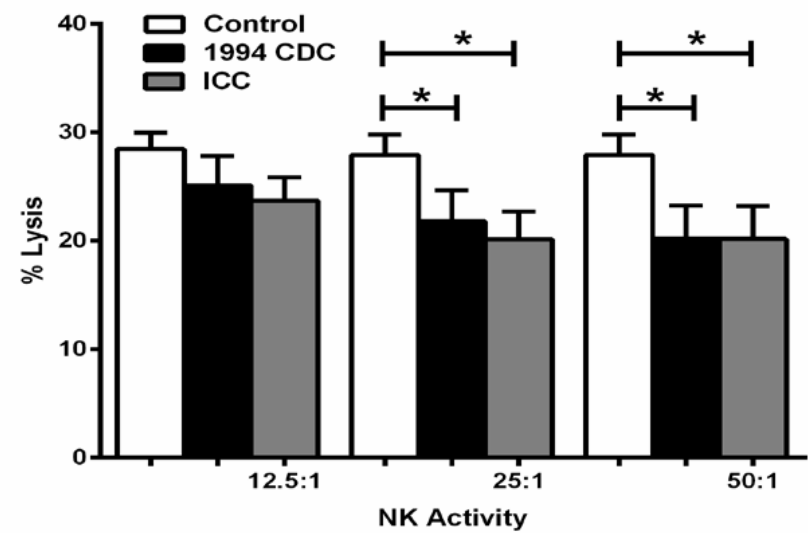

Figure 1: Comparison of NK cell cytotoxic activity in 1994 CDC and ICC CFS ME patients. A significant association was observed between CFS/ME cases and reduced NK cytotoxic activity. Data is represented as means \pm SEM. represents level of significance where P-values are less than 0.05 and the lines indicate significance between groups.

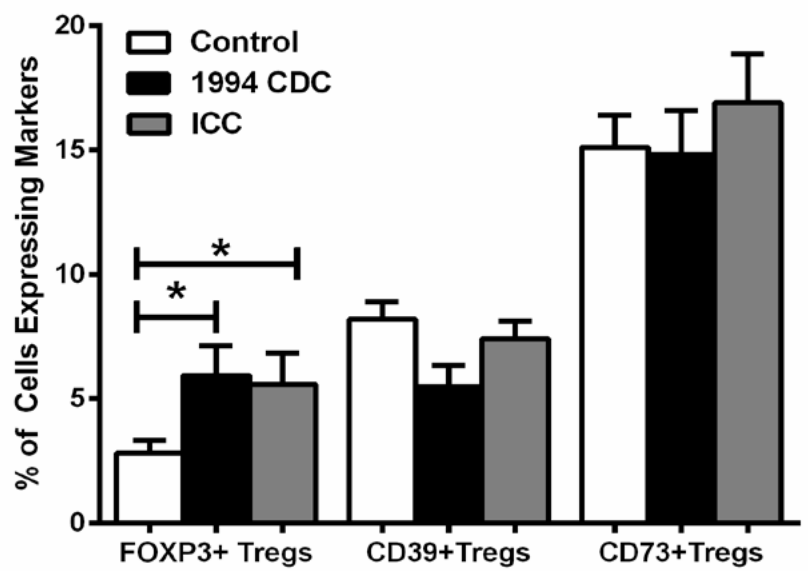

Figure 2: Treg markers are increased in both 1994 CDC and ICC CFS/ME patients. Monoclonal antibodies were used to examine the expression of $\mathrm{CD}^{+}$, $\mathrm{CD}^{+} 5^{+}, \mathrm{CD} 127^{+}, \mathrm{FOXP3}^{+}$Tregs and also the expression of CD39 and CD73 on Tregs in CFS/ME cases and non-fatigue controls. Data represents means \pm SEM. * represents level of significance where P-values are less than 0.05 and the lines indicate significance between groups. of 50.7, 49.3, and 49.7 years for 1994 CDC, ICC, healthy controls, respectively. $1994 \mathrm{CDC}$ and ICC patients also shared a similar duration of illness of 19 years.

\section{NK cell activity}

In both 1994 CDC and ICC patients, NK cell activity was significantly reduced in comparison to healthy controls. Measures of NK cell lytic proteins did not yield any significance difference between the groups (Figure 1).

\section{Tregs and neutrophil antigens}

A significant increase in Tregs was observed in both 1994 CDC and ICC patients in comparison to healthy controls (Figure 2). Further a significant decrease in CD39+ Tregs was observed in 1994 CDC patients in comparison to healthy controls. No Significant differences were observed in B cells, NK cells and DC phenotypes. HNA2 (CD177 ) were significantly increased in 1994 CDC patients while HNA2 $\left(\mathrm{CD} 177^{+}\right)$were significantly reduced in ICC patients in comparison to healthy controls (Figure 3). HNA5 was higher in 1994 CDC patients in comparison to ICC patients.

\section{NK KIR receptors}

Overall the expression of KIR receptors and NKG2D was higher in ICC patients in comparison with 1994 CDC patients. The ICC patients indicated significant elevations in the expression of KIR2DL1, KIR3DL1 and NKG2D compared to the 1994 CDC patients. KIR2DL2 was also significantly higher in ICC patients (Figure 4).

Correlation between immunological parameters with physical functioning

The results of significant correlation between immunological parameters with mean PCS, PF and $\mathrm{M}$ scores are shown in Table 1. Among healthy controls, higher PCS scores were significantly associated with higher NK cell activity. Among ICC patients, lower $\mathrm{CD} 9^{+}$Tregs was associated with lower PCS and PF scores on the SF-36 and higher mobility scores on the WHO DAS 2.0. A lower number of NK cells lacking KIR3DL1 scores were also associated with higher PCS scores and a higher expression of KIR2DS4 receptors. No significant associations at the $\mathrm{p}<0.001$ level were detected among 1994 CDC patients.

\section{Discussion}

This is the first study to examine whether immunological differences may be found among patients fulfilling different diagnostic criteria for CFS/ME, according to the 1994 CDC and ICC. The findings indicate that the ICC is invaluable in identifying immunological differences that have consistently been shown to be decreased in CFS/ME patients diagnosed with the 1994 CDC. The ICC is able to determine additional differences within immunological parameters that were not previously observed in 1994 CDC cases. In the present study, NK cell cytotoxic activity and HNA2 $\left(\mathrm{CD} 177^{+}\right)$expression were profoundly reduced, while Tregs and CD39+ Tregs were increased in the CFS/ME cases. Only HNA5 was significantly increased in the ICC group in comparison to the 1994 CDC group. These findings are highly suggestive of a need to incorporate both the 1994 CDC and ICC in future clinical research as it may allow for the identification of subgroups.

To date, the only available diagnostic tools for CFS/ME are symptom based criteria such as the 1994 CDC, and ICC. Universal consent on experimental findings and usage of these criteria may achieve and likely 


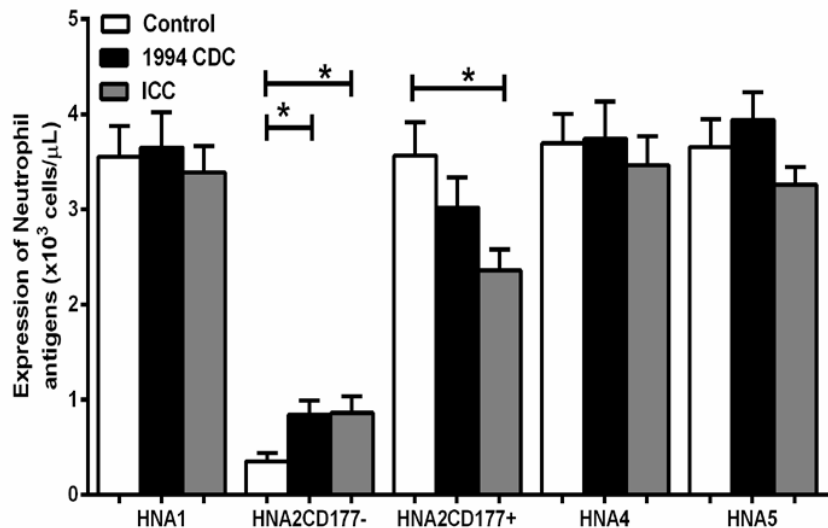

Figure 3: Assessment of HNA antigens in 1994 CDC and ICC CFS/ME patients. Expression of human neutrophil antigens HNA1, HNA2, HNA4 and HNA5 were examined in all participants following staining of whole blood samples with monoclonal antibodies specific for neutrophils. Data represents means \pm SEM * represents level of significance where P-values are less than 0.05 and the lines indicate significance between groups.

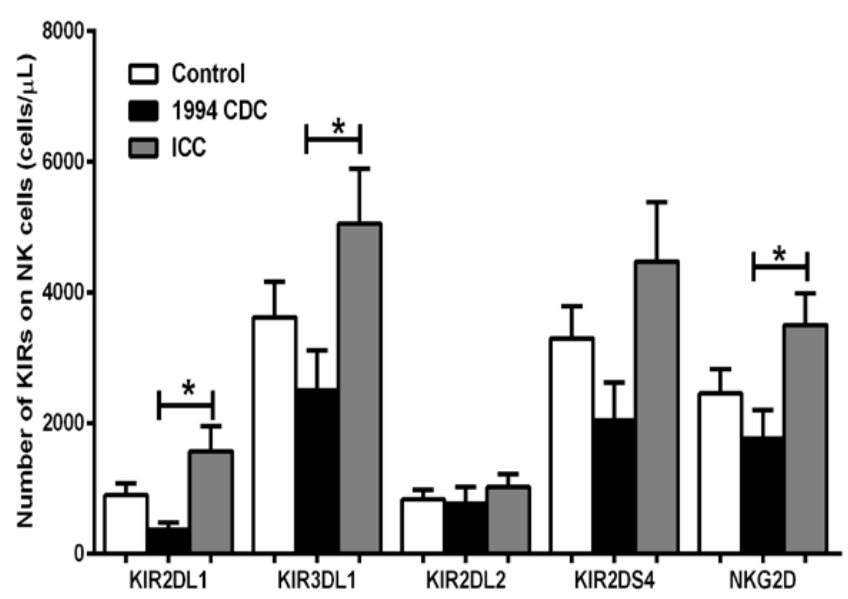

Figure 4: Distribution of KIR receptors in 1994 CDC and ICC CFS/ME patients Isolated NK cells were examined for the expression of NK receptors following labelling with different monoclonal antibodies specific for NK receptors. Data is represented as means \pm SEM. * represents level of significance where $P$-values are less than 0.05 and the lines indicate significance between groups.

improve the efficiency of producing clinical measurable diagnostic markers in the future. NK cell activity is a promising candidate for CFS/ME diagnostic marker. A global impairment in NK activity has consistently been observed in CFS/ME patients $[7,15,16,19,20]$. This suggests an inability of the NK cells in CFS/ME patients to clear viral and other microbial infections and also a prevalence of recurring viral infections. NK cells are essential to initial response to viruses and clearance of activated macrophages and autoreactive T cells [21]. Deficiencies in NK activity have been proposed to be secondary to alterations in perforin and granzymes, mediators of cytotoxic activity $[16,20]$. In the present study, reduced NK activity was not correlated with alterations in lytic proteins as these were not statistically different from the healthy controls. This may likely be attributed to the limitations of the statistical power due to the numbers of participants enrolled in the study.
In a recent study, NK cell KIR receptors were observed to be significantly different in CFS/ME with moderate and severe symptoms [19]. Similarly, in the present study, when patients were categorized as 1994 CDC and ICC, marked differences were noticed in the expression of NK receptors. Specifically, in the ICC group profound increases were observed in KIR2DL1, KIR3DL1 and NKG2D in contrast to the 1994 CDC group. KIR2DL1 and KIR3DL1 are inhibitory receptors while NKG2D is an activating receptor, these receptors inhibit and activate NK cytotoxic activity respectively [22-25]. An increase in inhibitory receptors in the ICC group may account for the reduced cytotoxic activity. Blocking inhibitory receptors such as KIR2DL1 has been shown to enrich NK cytotoxic activity cancer cell lines [26]. Pronounced expression of KIR3DL1 has been associated with the presentation of certain alleles including KIR3DL1 $1^{\star} 001,002,003$ and 008 while low levels are recognized in individuals expressing KIR3DL1 ${ }^{\star} 004,005,006$ and 007 alleles [27].

It is therefore possible to speculate that the CFS/ME patients in this study, especially those belonging to the ICC group may have expressed higher frequencies of certain KIR alleles, increasing the levels of inhibition on cytotoxic activity and depressing their ability to

\begin{tabular}{|c|c|c|c|}
\hline Immunological parameters & PCS & PF & $\mathbf{M}$ \\
\hline \multicolumn{4}{|l|}{ Perforin } \\
\hline \multicolumn{4}{|l|}{ GrzA } \\
\hline \multicolumn{4}{|l|}{ GrzB } \\
\hline NK activity & $\begin{array}{c}\text { Healthy Controls } \\
0.616 \\
p<0.001\end{array}$ & & \\
\hline \multicolumn{4}{|l|}{ Imm B cells } \\
\hline \multicolumn{4}{|l|}{ Memory B cells } \\
\hline \multicolumn{4}{|l|}{ Plasma B cells } \\
\hline \multicolumn{4}{|l|}{ CD56 dim } \\
\hline \multicolumn{4}{|l|}{ CD56 bright } \\
\hline \multicolumn{4}{|l|}{ Tregs } \\
\hline CD39+ Tregs & $\begin{array}{c}\text { ICC } \\
0.835 \\
p<0.001\end{array}$ & $\begin{array}{c}\text { ICC } \\
0.722 \\
p<0.001\end{array}$ & $\begin{array}{c}\text { ICC } \\
-0.794 \\
p<0.001\end{array}$ \\
\hline \multicolumn{4}{|l|}{$\mathrm{CD}^{+} 3^{+}$Tregs } \\
\hline \multicolumn{4}{|l|}{ HNA1 } \\
\hline \multicolumn{4}{|l|}{ HNA2 (CD177-) } \\
\hline \multicolumn{4}{|l|}{ HNA2 (CD177+) } \\
\hline \multicolumn{4}{|l|}{ HNA4 } \\
\hline \multicolumn{4}{|l|}{ HNA5 } \\
\hline \multicolumn{4}{|l|}{$\mathrm{mDCs}$} \\
\hline \multicolumn{4}{|l|}{ pDCs } \\
\hline \multicolumn{4}{|l|}{ CD16+ DCs } \\
\hline \multicolumn{4}{|l|}{ KIR2DL1 neg } \\
\hline \multicolumn{4}{|l|}{ KIR2DL1 pos } \\
\hline KIR3DL1 neg & $\begin{array}{c}\text { ICC } \\
-0.693 \\
p<0.001\end{array}$ & & \\
\hline \multicolumn{4}{|l|}{ KIR3DL1 pos } \\
\hline \multicolumn{4}{|l|}{ KIR2DL2 neg } \\
\hline \multicolumn{4}{|l|}{ KIR2DL2 pos } \\
\hline \multicolumn{4}{|l|}{ KIR2DS4 neg } \\
\hline KIR2DS4 pos & $\begin{array}{c}\text { ICC } \\
-0.678 \\
p<0.001\end{array}$ & & \\
\hline \multicolumn{4}{|l|}{ NKG2D neg } \\
\hline NKG2Dp pos & & & \\
\hline
\end{tabular}

Table 1: Correlations between immunological parameters and physical functioning scores. 
Citation: Brenu EW, Johnston S, Hardcastle SL, Huth TK, Fuller K, et al. (2013) Immune Abnormalities in Patients Meeting New Diagnostic Criteria for Chronic Fatigue Syndrome/Myalgic Encephalomyelitis. J Mol Biomark Diagn 4: 152. doi:10.4172/2155-9929.1000152

effectively clear infections. Hence, the ICC may be useful in predicting subgroups of patients based on certain molecular markers and this may be important in the clinical diagnosis of CFS/ME. This is the first time an increase in NKG2D receptors have been reported in CFS/ME patients. In an attempt to restore balance between activating and inhibitory signals, this may likely have ensued to counteract the overwhelming inhibitory signals emanating from the KIR2DL1 and KIR3DL1 [28]. These findings presuppose that differing mechanisms may be responsible for reduced cytotoxic activity in CFS/ME patients.

Increased Tregs is emerging as an important marker of CFS/ME. To date recent studies have indicated abnormal elevations of Tregs in CFS/ ME patients in comparison to healthy controls [16,29]. Most of these Tregs expressed the transcription factor Foxp3. This Treg dysfunction may be an added factor that limits the magnitude of NK activity further weakening the already diminished cytotoxic activity in CFS/ME. The abnormal levels of Tregs did not differ between the two cases of CFS/ ME. Interestingly, in the 1994 CDC cohort increased levels of Tregs occurred in conjunction with an overwhelmingly decrease in CD39. CD39 is known to convert extracellular ATP into AMP and its decrease may cause exacerbations in pro-inflammatory immune responses [30] and consequently $\mathrm{T}$ cell responses [31]. It is not known whether these effects occur in CFS/ME patients.

Previous findings have alluded to increased levels of CD73 in CFS/ ME patients diagnosed using the 1994 CDC criteria [32], in the current study a clear increased in CD73 was observed in both groups but this did not achieve statistical significance. CD73 converts AMP to adenosine, regulating the immune response [33]. Abnormal levels in these parameters may alter inflammatory reactions in the CFS/ME patients altering the extent of Treg suppression of other immune cells and immune processes [31]. Neutrophil dysfunction has received limited investigation is CFS/ME. Ultimately, perceived decreases in neutrophil respiratory burst and differential expression in HNA antigens highlight substantial deficits in the neutrophil function $[15,32]$.

An important question remains on the relationship between immunological perturbations and physical functioning. This study examined whether these associations could be identified, using simple, validated measures of physical status [34-37] and is the first to examine this using these immune parameters. Among healthy controls, lower NK cell activity was associated with poorer physical status. Though this was not observed among CFS/ME patients, the findings to support that lower NK cell activity in general may be a sign of poor health. As CFS/ ME patients are shown to have significantly lower NK cell activity than healthy controls, this may continue to be a reliable indicator of their illness.

No strong associations were observed among 1994 CDC patients however, several were found among ICC patients. Though CD $39^{+}$was shown to be significantly decreased among 1994 CDC patients, it was not indicative of this groups physical status. Interestingly, decreased CD39+ was strongly associated with poorer physical health across all scores used in this study among ICC patients. Significantly increased KIR3DL1 and decreased KIR3Dl1 among ICC patients were also strongly associated with poorer physical health scores. These findings may be reflective of the groups poorer physical status overall. In previous investigations, ICC patients have been identified as an important subgroup within CFS/ME with greater impairment according to the scores used in this study $[10,11]$.

On the basis of this present study the features that distinguish patients selected using the ICC from the 1994 CDC are KIR receptors and HNA5. It is not clear why CD39 was reduced in the 1994 CDC group in relation to healthy controls. The absence of changes in other cell phenotypes and markers assessed in this study may be related to the heterogeneity of the CFS/ME. This also indicates that CFS/ME is not necessarily associated with irregular distribution of lymphocyte subtypes but rather deregulation of intricate functional capacities of these immune lymphocytes. It is important to consider in the interpretation of the findings that a relationship between immunological parameters and physical health does not imply causality but supports the hypothesis that some CFS/ME patients have an underlying immune mechanism contributing to their illness. Since not all CFS/ME patients have identifiable immune abnormalities, and it may be useful in future assessment to differentiate subgroups of patients according to more specific clinical presentations such as symptom clusters, severity, frequency and duration of illness. Though not possible with the current participant numbers, this approach may serve to decrease heterogeneity observed in this illness. Further research is therefore needed to establish the significance of these immunological abnormalities and the influence of further clinical measures, which will aid in future diagnosis of this illness.

\section{Acknowledgment}

This research was funded and supported by the Mason Foundation (grant number 43120) and the Alison Hunter Memorial Foundation (grant number HF 201).

\section{References}

1. Carruthers BM, Jain AK, De Meirleir KL, Peterson DL, Klimas NG, et al. (2003) Myalgic Encephalomyelitis/Chronic Fatigue Syndrome: Clinical Working Case Definition, Diagnostic and Treatment Protocols. Journal of Chronic Fatigue Syndrome 11: 7-154

2. Fukuda K, Straus SE, Hickie I, Sharpe MC, Dobbins JG, et al. (1994) The chronic fatigue syndrome: A comprehensive approach to its definition and study. International Chronic Fatigue Syndrome Study Group. Ann Intern Med 121: 953-959.

3. Lloyd AR, Hickie I, Boughton CR, Spencer O, Wakefield D (1990) Prevalence of chronic fatigue syndrome in an Australian population. Med J Aust 153: 522-528.

4. Sharpe MC, Archard LC, Banatvala JE, Borysiewicz LK, Clare AW, et al. (1991) A report--chronic fatigue syndrome: guidelines for research. J R Soc Med 84 118-121.

5. Carruthers BM, van de Sande MI, De Meirleir KL, Klimas NG, Broderick G, et al. (2011) Myalgic encephalomyelitis: International Consensus Criteria. J Intern Med 270: 327-338.

6. Holmes GP, Kaplan JE, Gantz NM, Komaroff AL, Schonberger LB, et al. (1988) Chronic fatigue syndrome: a working case definition. Ann Intern Med 108: 387 389.

7. Brenu EW, van Driel ML, Staines DR, Ashton KJ, Hardcastle SL, et al. (2012) Longitudinal investigation of natural killer cells and cytokines in chronic fatigue syndrome/myalgic encephalomyelitis. J Transl Med 10: 88

8. Jason LA, Coffadi K, Torres-Harding S (2007) Toward an empirical case definition of CFS. Journal of Social Service Research 34: 43-54.

9. Kennedy G, Abbot NC, Spence V, Underwood C, Belch JJ (2004) The specificity of the CDC-1994 criteria for chronic fatigue syndrome: comparison of health status in three groups of patients who fulfill the criteria. Ann Epidemiol 14: 95-100.

10. Jason LA, Brown A, Evans M, Sunnquist M, Newton JL (2013) Contrasting Chronic Fatigue Syndrome versus Myalgic Encephalomyelitis/Chronic Fatigue Syndrome. Fatigue 1: 168-183.

11. Johnston S, Brenu EW, Hardcastle S, Huth T, Fuller K, et al. (2013) Physical and social functioning in varying cases of Chronic Fatigue Syndrome/Myalgic Encephalomyelitis. In review.

12. Ware JE Jr, Sherbourne CD (1992) The MOS 36-item short-form health survey (SF-36). I. Conceptual framework and item selection. Med Care 30: 473-483. 
Citation: Brenu EW, Johnston S, Hardcastle SL, Huth TK, Fuller K, et al. (2013) Immune Abnormalities in Patients Meeting New Diagnostic Criteria for Chronic Fatigue Syndrome/Myalgic Encephalomyelitis. J Mol Biomark Diagn 4: 152. doi:10.4172/2155-9929.1000152

13. World Health Organisation (2001) WHO Disability Adjustment Schedule II (WHODAS II).

14. Aubry JP, Blaecke A, Lecoanet-Henchoz S, Jeannin P, Herbault N, et al. (1999) Annexin $V$ used for measuring apoptosis in the early events of cellular cytotoxicity. Cytometry 37: 197-204.

15. Brenu EW, Staines DR, Baskurt OK, Ashton KJ, Ramos SB, et al. (2010) Immune and hemorheological changes in chronic fatigue syndrome. J Trans Med 8: 1.

16. Brenu EW, van Driel ML, Staines DR, Ashton KJ, Ramos SB, et al. (2011) Immunological abnormalities as potential biomarkers in Chronic Fatigue Syndrome/Myalgic Encephalomyelitis. J Transl Med 9: 81.

17. Anane LH, Edwards KM, Burns VE, Zanten JJ, Drayson MT, et al. (2010) Phenotypic characterization of gammadelta $T$ cells mobilized in response to acute psychological stress. Brain Behav Immun 24: 608-614.

18. Alter G, Malenfant JM, Altfeld M (2004) CD107a as a functional marker for the identification of natural killer cell activity. J Immunol Methods 294: 15-22.

19. Brenu EW, Hardcastle SL, Atkinson GM, Driel ML, Kreijkamp-Kaspers S, et al. (2013) Natural killer cells in patients with severe chronic fatigue syndrome. Autoimmunity Highlights: 1-12.

20. Maher KJ, Klimas NG, Fletcher MA (2005) Chronic fatigue syndrome is associated with diminished intracellular perforin. Clin Exp Immunol 142: 505511.

21. Flodström-Tullberg M, Bryceson YT, Shi FD, Höglund P, Ljunggren HG (2009) Natural killer cells in human autoimmunity. Curr Opin Immunol 21: 634-640.

22. Yawata M, Yawata N, Draghi M, Little AM, Partheniou F, et al. (2006) Roles for HLA and KIR polymorphisms in natural killer cell repertoire selection and modulation of effector function. J Exp Med 203: 633-645.

23. Rajalingam R (2012) Overview of the killer cell immunoglobulin-like receptor system. Methods Mol Biol 882: 391-414.

24. Vojvodic S, Popovic S (2010) [Natural killer cells--biology, functions and clinical relevance]. Med Pregl 63: 91-97.

25. Jamil KM, Khakoo SI (2011) KIR/HLA interactions and pathogen immunity. J Biomed Biotechnol 2011: 298348

26. Wu GQ, Zhao YM, Huang H, Lai XY (2011) [Effects of blocking inhibitory KIR receptors on cytotoxic activity of human NK cells in vitro]. Zhejiang Da Xue Xue Bao Yi Xue Ban 40: 475-481.
27. Gardiner CM, Guethlein LA, Shilling HG, Pando M, Carr WH, et al. (2001) Different NK cell surface phenotypes defined by the DX9 antibody are due to KIR3DL1 gene polymorphism. J Immunol 166: 2992-3001.

28. Decot V, Voillard L, Latger-Cannard V, Aissi-Rothé L, Perrier P, et al. (2010) Natural-killer cell amplification for adoptive leukemia relapse immunotherapy: comparison of three cytokines, IL-2, IL-15, or IL-7 and impact on NKG2D KIR2DL1, and KIR2DL2 expression. Exp Hematol 38: 351-362.

29. Curriu M, Carrillo J, Massanella M, Rigau J, Alegre J, et al. (2013) Screening NK-, B- and T-cell phenotype and function in patients suffering from Chronic Fatigue Syndrome. J Transl Med 11: 68

30. Borsellino G, Kleinewietfeld M, Di Mitri D, Sternjak A, Diamantini A, et al. (2007) Expression of ectonucleotidase CD39 by Foxp3+ Treg cells: hydrolysis of extracellular ATP and immune suppression. Blood 110: 1225-1232.

31. Sauer AV, Brigida I, Carriglio N, Hernandez RJ, Scaramuzza S, et al. (2012) Alterations in the adenosine metabolism and CD39/CD73 adenosinergic machinery cause loss of Treg cell function and autoimmunity in ADA-deficien SCID. Blood 119: 1428-1439.

32. Brenu EW, Huth TK, Hardcastle SL, Fuller K, Kaur M, et al. (2013) The Role of Adaptive and Innate Immune Cells in Chronic Fatigue Syndrome/ Myalgic Encephalomyelitis. In review.

33. Romio M, Reinbeck B, Bongardt S, Hüls S, Burghoff S, et al. (2011) Extracellula purine metabolism and signaling of CD73-derived adenosine in murine Treg and Teff cells. Am J Physiol Cell Physiol 301: C530-539.

34. Buchwald D, Pearlman T, Umali J, Schmaling K, Katon W (1996) Functional status in patients with chronic fatigue syndrome, other fatiguing illnesses, and healthy individuals. Am J Med 101: 364-370.

35. Garin O, Ayuso-Mateos JL, Almansa J, Nieto M, Chatterji S, et al. (2010) Validation of the "World Health Organization Disability Assessment Schedule, WHODAS-2" in patients with chronic diseases. Health Qual Life Outcomes 8: 51.

36. Hardt J, Buchwald D, Wilks D, Sharpe M, Nix WA, et al. (2001) Health-related quality of life in patients with chronic fatigue syndrome: an international study. J Psychosom Res 51: 431-434.

37. Komaroff AL, Fagioli LR, Doolittle TH, Gandek B, Gleit MA et al. (1996) Health status in patients with chronic fatigue syndrome and in general population and disease comparison groups. Am J Med 101: 281-290.
Citation: Brenu EW, Johnston S, Hardcastle SL, Huth TK, Fuller K, et al (2013) Immune Abnormalities in Patients Meeting New Diagnostic Criteria for Chronic Fatigue Syndrome/Myalgic Encephalomyelitis. J Mol Biomark Diagn 4 : 152. doi:10.4172/2155-9929.1000152

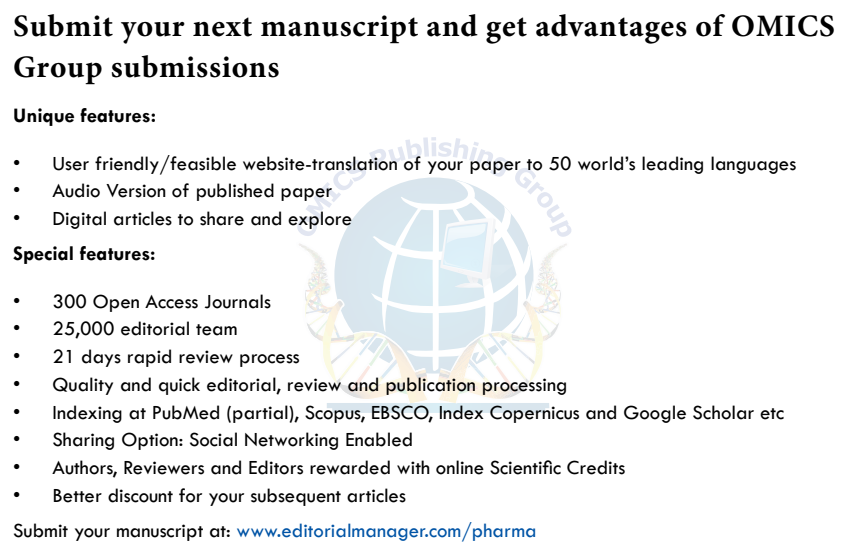

Submit your next manuscript and get advantages of OMICS Group submissions

Unique features:

User friendly/feasible website-translation of your paper to 50 world's leading languages

Audio Version of published paper

- Digital articles to share and explore

Special features:

300 Open Access Journals

25,000 editorial team

- 21 days rapid review process

Quality and quick editorial, review and publication processing

- Indexing at PubMed (partial), Scopus, EBSCO, Index Copernicus and Google Scholar etc

- Sharing Option: Social Networking Enabled

- Authors, Reviewers and Editors rewarded with online Scientific Credits

Better discount for your subsequent articles

Submit your manuscript at: www.editorialmanager.com/pharma 